\title{
Discovery of differentially expressed genes in cashmere goat (Capra hircus) hair follicles by RNA sequencing
}

X. Qiao ${ }^{1 *}$, J.H. Wu ${ }^{2,3,4 *}$, R.B. Wu ${ }^{5 *}$, R. Su${ }^{1,4}, C^{2}$ Li $^{6}$, Y.J. Zhang ${ }^{1}$, R.J. Wang ${ }^{1}$, Y.H. Zhao ${ }^{1}$, Y.X. Fan ${ }^{1}$, W.G. Zhang ${ }^{1}$ and J.Q. Li ${ }^{1}$

${ }^{1}$ College of Animal Science, Inner Mongolia Agricultural University, Hohhot, Inner Mongolia, China

${ }^{2}$ Inner Mongolia Prataculture Research Center, Chinese Academy of Science, Hohhot, China

${ }^{3}$ Animal Husbandry Institute,

Inner Mongolia Academy of Agricultural and Animal Husbandry Sciences, Hohhot, China

${ }^{4}$ State Key Laboratory of Genetic Resources and Evolution, Kunming Institute of Zoology, Chinese Academy of Sciences, Kunming, China

${ }^{5}$ College of Basic Medical Sciences, Inner Mongolia Medical University,

Hohhot, China

${ }^{6}$ College of Animal Science and Technology,

Inner Mongolia University for Nationalities, Tongliao, Inner Mongolia, China

*These authors contributed equally to this study.

Corresponding authors: W.G. Zhang / R. Su

E-mail: atcgnmbi@aliyun.com / suruiyu@126.com

Genet. Mol. Res. 15 (3): gmr.15038589

Received March 1, 2016

Accepted June 14, 2016

Published September 2, 2016

DOI http://dx.doi.org/10.4238/gmr.15038589

Copyright (C) 2016 The Authors. This is an open-access article distributed under the terms of the Creative Commons Attribution ShareAlike (CC BY-SA) 4.0 License.

ABSTRACT. The mammalian hair follicle (HF) is a unique, highly regenerative organ with a distinct developmental cycle. Cashmere goat 
(Capra hircus) HFs can be divided into two categories based on structure and development time: primary and secondary follicles. To identify differentially expressed genes (DEGs) in the primary and secondary HFs of cashmere goats, the RNA sequencing of six individuals from Arbas, Inner Mongolia, was performed. A total of 617 DEGs were identified; 297 were upregulated while 320 were downregulated. Gene ontology analysis revealed that the main functions of the upregulated genes were electron transport, respiratory electron transport, mitochondrial electron transport, and gene expression. The downregulated genes were mainly involved in cell autophagy, protein complexes, neutrophil aggregation, and bacterial fungal defense reactions. According to the Kyoto Encyclopedia of Genes and Genomes database, these genes are mainly involved in the metabolism of cysteine and methionine, RNA polymerization, and the MAPK signaling pathway, and were enriched in primary follicles. A microRNA-target network revealed that secondary follicles are involved in several important biological processes, such as the synthesis of keratin-associated proteins and enzymes involved in amino acid biosynthesis. In summary, these findings will increase our understanding of the complex molecular mechanisms of HF development and cycling, and provide a basis for the further study of the genes and functions of HF development.

Key words: Cashmere goat; Differentially expressed gene; Hair follicle; RNA-Seq; Whole transcriptome sequencing

\section{INTRODUCTION}

Cashmere originated in central Asia and is derived from the lightweight under hair of cashmere goats (Capra hircus). Cashmere is an important economic product, and the quantity and quality of cashmere mainly depend on hair follicle (HF) characteristics (Berger et al., 2015; Wang et al., 2015). The Inner Mongolia Autonomous Region of China produces over $40 \%$ of the world's total cashmere, and cashmere is an important component of the local agricultural economy. The Inner Mongolia cashmere goat is a dual-purpose breed with a long history. It adapts well to desert and semi-desert pastures, and has a fine, soft, downy, winter undercoat (Luikart et al., 2001; Keva, 2005). Because it can produce a large amount of high-quality cashmere fiber, the cashmere goat has attracted the attention of many researchers in recent years. The development of cashmere is a complicated process in which genetic factors, as well as environmental factors, may play an important role. Little is known about the molecular mechanism involved in cashmere formation and development, particularly the different contributions of the two kinds of HF. Identifying the genes involved in HF development and cycling may reveal the regulation mechanism underlying cashmere development.

Mammalian skin contains thousands of HFs, which produce hair for thermoregulation, protection against environmental trauma, information collection, and social communication (Stenn and Paus, 2001; Botchkarev and Paus, 2003). HF development occurs as a result of extensive epithelial-mesenchymal interactions (Botchkarev and Kishimoto, 2003; Andl et al., 2004). Morphologically, the process can be divided into three distinct stages: induction,

Genetics and Molecular Research 15 (3): gmr.15038589 
organogenesis, and cytodifferentiation (Schmidt-Ullrich and Paus, 2005). The histological structures of mature HFs consist of the hair bulb, inner root sheath, hair shaft, outer root sheath, and connective tissue sheath. There are two kinds of HF: primary and secondary follicles. Primary follicles produce guard hair that is characteristically medullated and coarse $(>30 \mu \mathrm{m})$ and provides mechanical protection. Secondary follicles are more numerous than primary follicles and produce nonmedullated, fine cashmere fiber $(<24 \mu \mathrm{m})$ that provides thermal protection (Ryder, 1967, 1970; Nixon et al., 1991; Dong et al., 2013). Cashmere is comprised of down and guard hair. The down, which is called cashmere, is produced by secondary follicles in three stages: anagen, catagen, and telogen. Guard hair is produced by the primary follicles. The thickening and elongation of epidermal keratinocytes (hair placodes) are the main processes in anagen HFs (Schneider et al., 2009). Although there is periodic variation in primary follicle activity (Nixon et al., 1991), primary follicles are relatively stable compared to secondary follicles. Within a complete growth cycle, secondary follicles are active for 10 months, while primary follicle activity may last longer. HF activity may affect the quality and yield of cashmere. Therefore, in this study, we aimed to find differentially expressed genes (DEGs) between primary and secondary follicles, in order to further our understanding of HF production and growth cycles.

The transcriptome is the complete set of transcripts in a cell, both in terms of type and quantity. With the development of next-generation sequencing technology, RNA sequencing (RNA-Seq) has become increasingly popular in transcriptome studies, due to its precision, speed, and sensitivity. Transcriptome studies catalog different kinds of RNA in an organism, and quantify changes in the expression levels of individual transcripts with time or under certain conditions (Marioni et al., 2008; Wang et al., 2009). The analysis of large volumes of RNA-Seq data has revealed novel isoforms and splice variants, as well as unannotated transcripts, even in well-studied biological models (Trapnell et al., 2010). The transcriptome reveals a great deal about the functional aspects of the genome, as well as the different kinds of biomolecules present within the cell or tissue, so is very useful for studying the genetics behind growth, development, and disease (Ghosh and Chan, 2016). To understand the mechanisms of gene regulation in these two follicles, we studied microRNAs (miRNAs) and their targets, which play important roles in the activation of secondary follicles. miRNAs are non-coding RNAs that fine-tune the regulation of gene expression at the transcriptional and post-transcriptional levels during ontogenetic development, metabolism, and disease. Recent studies have demonstrated that miRNAs are associated with maintaining multipotency in mammalian embryos, suggesting that they may also be involved in cashmere production. We used RNA-Seq technology to investigate the whole transcriptomes of two kinds of goat follicles, and obtain a large number of functional genes involved in HF identity and cashmere production. In addition, by investigating miRNA targets in each follicle, we found that miRNAs play an important role in the regulation of HF development and activity. These findings provide useful information on secondary HF development and can be used in future studies.

\section{MATERIAL AND METHODS}

\section{Animals and sample collection}

Inner Mongolia cashmere goats obtained from a goat stud farm (Erdos, Inner Mongolia) were used. All of the samples were taken under supervision. Six 3-year-old, female goats with a similar nutritional status were randomly selected. Hair and cashmere samples were plucked

Genetics and Molecular Research 15 (3): gmr.15038589 
and isolated from the sides of the selected goats. Primary and secondary HFs were harvested from hair and cashmere roots, respectively, by skilled technicians, frozen in liquid nitrogen, and stored at $-80^{\circ} \mathrm{C}$ until analysis.

\section{RNA extraction and quality analysis}

Total RNA was extracted from the primary and secondary HFs using an RNAprep Pure Kit (Tiangen, Beijing) according to the manufacturer instructions. Upon extraction, the RNA was quantified using a NanoDrop ${ }^{\mathrm{TM}} 2000$ spectrophotometer (Thermo Fisher Scientific, USA), and $5 \mu \mathrm{L}$ was resolved on $1.0 \%(\mathrm{w} / \mathrm{v})$ agarose gel for quality assessment.

\section{Ethics statement}

All of the animal procedures were approved by the Inner Mongolia Agriculture University Animal Care and Use Committee in accordance with the National Animal Care Standard (GB 14925-2001). All efforts were made to minimize animal suffering.

\section{Quality control}

The raw sequencing data were evaluated by FastQC (Babraham Bioinformatics), and included the quality distribution of nucleotides, position-specific sequencing quality, GC content, the proportion of polymerase chain reaction duplications, and k-mer frequencies.

\section{Mapping}

We used TopHat (https://ccb.jhu.edu/software/tophat/index.shtml) (Trapnell et al., 2009), which is a fast splice junction mapper for RNA-Seq reads, for the RNA-Seq alignment. After aligning the reads to the reference genome based on Bowtie (http:// sourceforge.net/projects/bowtie-bio/files/bowtie2/2.1.0/) (Langmead et al., 2009), the mapped reads were assembled using Maq (http://maq.sourceforge.net/) to identify possible splicing junctions. Unmapped reads were divided into small segments in order to allow them to align to the reference genome (hg19 and mm10), and define splice junctions by the seed-extension procedure.

\section{Expression profiling and analysis of differential expression}

Two sets of gene expression profiles were generated by RNA-Seq. To identify DEGs between the primary and secondary follicle samples, we first normalized the expression score using an upper-quartile algorithm to avoid the error caused by lowabundance genes, and then determined the DEGs between the two types of HF using the DEGSeq package (Bullard et al., 2010).

\section{Gene Ontology (GO) analysis}

GO analysis was used to analyze the main functions of the DEGs (Ashburner et al., 2000).

Genetics and Molecular Research 15 (3): gmr.15038589 


\section{Statistical analysis}

Pathway analysis was used to ascertain the main pathways of the DEGs. Pathway annotations of microarray genes were downloaded from the Kyoto Encyclopedia of Genes and Genomes (KEGG) database (http://www.genome.jp/kegg/). The Fisher exact test was used to find significant enrichment pathways, and the resulting $\mathrm{P}$ values were adjusted using the false discovery rate (FDR) algorithm (Benjamini and Hochberg, 1995). Pathway categories with an FDR of less than 0.05 are reported.

\section{Construction of the miRNA-target network}

Through the analysis of the function and signal transduction pathway of the important target genes regulated by miRNAs, we constructed a miRNA-target network combining miRNAs with its corresponding target genes, and obtained the most important miRNAs and its target genes by the method of graph theory.

\section{RESULTS}

\section{RNA-Seq of primary and secondary HFs and alignment with the reference genome}

After discarding sequences shorter than $18 \mathrm{nt}$, low-quality sequences, and contaminants formed by adapter-adapter ligation, reads without 3 ' ligation and insert tags were obtained in triplicate using FastQC. In total, 16,346,242, 22,659,001, and 20,003,308 clean reads were obtained for the primary HFs and 23,864,273, 24,945,508, and 20,203,387 clean reads were obtained for the secondary HFs. We then analyzed the quality scores and GC contents based on the triplicate libraries and distinct sequences to assess the sequencing quality (Figure 1A). All of the reads were approximately $50 \% \mathrm{GC}$, and the GC count per read was consistent with the theoretical GC distribution (Figure 1B). Subsequently, in order to analyze their expression and distribution in the goat genome, all of the clean Solexa reads were aligned with the goat genome sequence using TopHat. To investigate gene function, we BLAST-searched the mapped genes to both the human (hg19) and mouse genomes (mm10) for gene annotation.

\section{Identification of DEGs between the primary and secondary follicles}

A total 617 DEGs were identified between the primary and secondary follicles (logFC $>0.585$ or $\log \mathrm{FC}<-0.585, \mathrm{P}<0.05)$. There were 297 highly expressed genes in the secondary follicles and 320 in the primary follicles. Among the highly expressed genes in the primary follicles, peptidylprolyl isomerase H (GOAT_ENSBTAP00000006026), peptidylprolyl isomerase-like 3 (GOAT_ENSBTAP00000007126), and retinol binding protein 4 (GOAT ENSBTAP00000000566) are related to retinoic acid biosynthesis/functions, and can regulate hair growth and the hair life cycle through Wnt signaling (Lefebvre et al., 1993; Viallet and Dhouailly, 1994). Notably, 17 of the 20 keratin and keratin-associated proteins were more highly expressed in the primary follicles than the secondary follicles, indicating that keratin and keratin-associated proteins may be responsible for forming the rigid hair shaft and altering the hair structure and diameter of the primary follicle. In the secondary follicles, genes involving transcription activation (GOAT_ENSBTAP00000053726), translational initiation 
(GOAT_ENSP00000364119, GOAT_ENSP00000253039, and GOAT_ENSP00000261868), and oxidation-reduction reactions (GOAT_ENSBTAP00000044670, GÖAT_ENSBTAP0000 0051891, GOAT_ENSBTAP00000027191, GOAT_ENSBTAP000000236 $\overline{6} 8$, and GOAT ENSP0000030068 8 ) were highly expressed, suggesting that secondary follicles are involved in more biological activity than primary follicles. These DEGs characterized the molecular identities of the two kinds of follicle.



B.

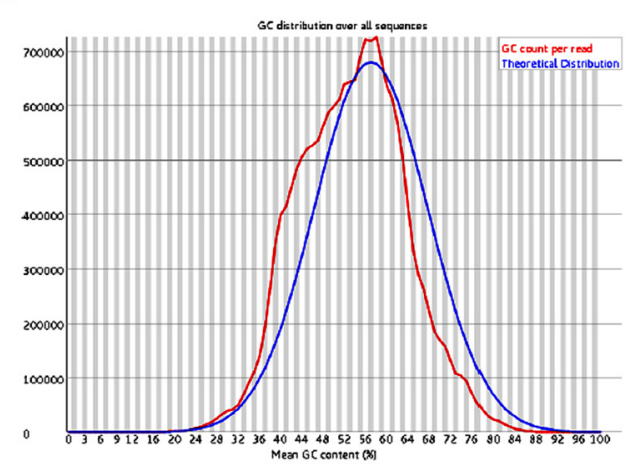

Figure 1. Quality control of single-pair RNA-Seq. A. Per-sequence quality scores. Horizontal axis represents the number of base pairs or range of base pairs; quality scores $>20$ indicate that the mapping rate was over $99 \%$. B. GC content over all of the sequences; red and blue lines represent actual GC content distribution and theoretical GC content distribution, respectively.

\section{Clustering primary and secondary follicle DEGs using GO and pathway analysis}

Based on their sequence homology, 617 highly expressed DEGs were categorized into 15 functional groups in the primary follicles and 13 in the secondary follicles, and the goat genes were aligned to the human genome (hg19) with $\mathrm{P}<0.01$. As shown in Figure $2 \mathrm{~A}$ and $2 \mathrm{~B}$, genes involved in inflammatory responses, immune activation, chemokine production, and chemotaxis were highly expressed in the primary follicles, and genes related to expression activation, translational initiation, positive regulation of DNA repair, protein K11-linked ubiquitination, and metabolic processes were highly expressed in the secondary follicles. Genes involved in protein K11-linked ubiquitination, which is related to keratin and keratin-

Genetics and Molecular Research 15 (3): gmr.15038589 
associated proteins, were highly expressed in the primary follicles. The KEGG pathway annotation revealed that the 617 DEGs were involved in 31 biological functions (Figure 3A and 3B). Most of the genes were involved in cellular metabolism, disease, and signal transduction. The most commonly indicated pathways were the disease and cancer-related pathways with 73 genes, representing $33.03 \%$ of the total DEGs, followed by the metabolic $(23.98 \%)$, mRNA and protein biosynthesis $(15.87 \%)$, and signal transduction pathways $(10.4 \%)$, indicating distinct biological differences between the primary and secondary HFs (Figure 3C).

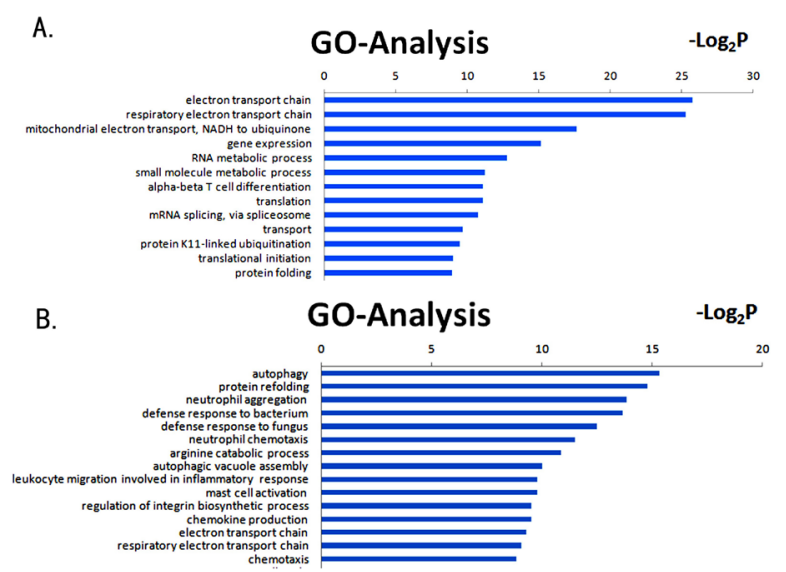

Figure 2. Gene ontology (GO) analysis of differentially expressed genes in primary and secondary follicles. A. Highly expressed genes in secondary follicles. B. Highly expressed genes in primary follicles.
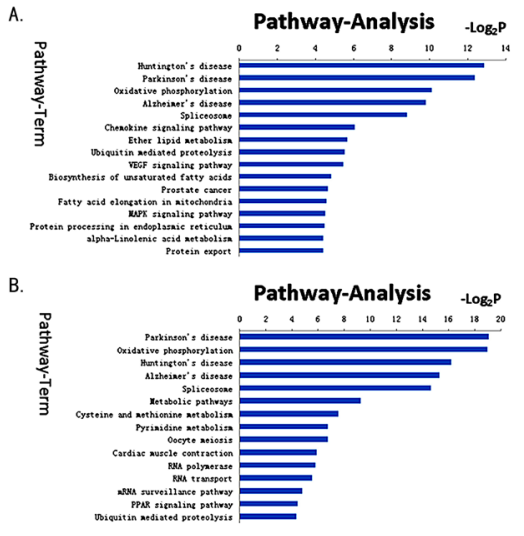

C.

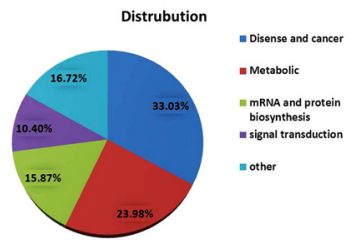

Figure 3. Pathway enrichment of differentially expressed genes in primary and secondary follicles. A. Pathways enriched with highly expressed genes in secondary follicles. B. Pathways enriched with highly expressed genes in primary follicles. C. Functional distribution of 617 differentially expressed genes in primary and secondary follicles. 


\section{Construction of the miRNA-target network}

A miRNA-target network was constructed based on the miRNA-mRNA interaction principle in the DEGs, and gene names were converted to the corresponding human gene symbols (Figure 4). Genes involved in important biological processes are regulated by miRNAs.

A.

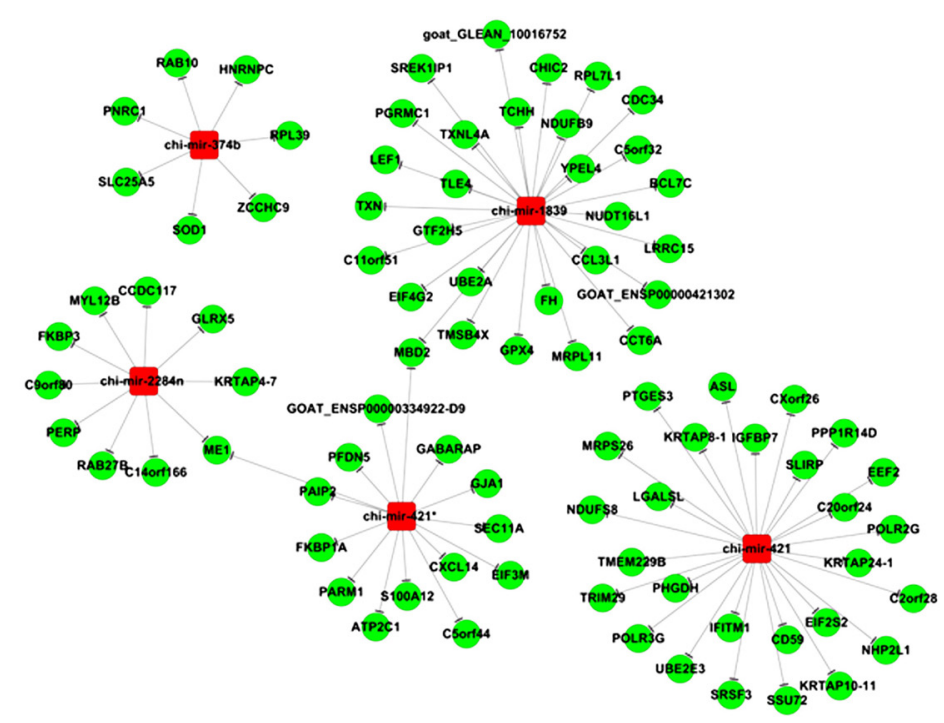

Figure 4. MicroRNA-target network. MicroRNAs are indicated in red and targets in green.

Keratin-associated proteins contribute to cashmere development, and keratin and keratin-associated proteins are the main structural proteins of hair fibers, and determine fiber quality. We annotated 86 keratin and keratin-associated protein genes in the goat follicle transcriptomes, 20 of which were differentially expressed between the primary and secondary follicles. Four keratin-associated protein genes that were detected in both types of follicle were targets of chi-mir-421 and chi-mir-2284n, and four genes responsible for translation initiation and elongation (EIF4G2, EIF3M, EIF2S2, and EEF2) were targets of chi-mir-1839, chi-mir-421*, and chi-mir-421. Other genes regulated by the five miRNAs were involved in oxidation-reduction reactions, the electron transport chain, and mitochondrial activity. These findings demonstrate that miRNA regulation plays an important role in controlling the activity of goat HFs. The miRNA-target network characterized the goat HF genes, and further analysis of these expression data may reveal the molecular foundation of cashmere fiber production.

\section{DISCUSSION}

Cashmere production is controlled by many genetic and physiological factors, such as the number of secondary follicles, ratio of primary to secondary follicles, and key regulators in cell cycle regulation (Rogers and Bawden, 2009). Primary HFs produce the coarse hair that is found in most mammals, and secondary HFs produce cashmere or fine hair in certain

Genetics and Molecular Research 15 (3): gmr.15038589 
mammals, including goats and antelopes (Ibraheem et al., 1994). Primary follicles have a sebaceous gland, but secondary follicles do not. Although many studies have found that transduction pathways such as Wnt, Shh, and Notch, and many growth factors, are involved in the regulation of follicle development, the molecular differences between the two kinds of follicle are still not fully understood.

We investigated the whole transcriptomes of goat primary HFs and secondary HFs using RNA technology, and generated a large number of reliable reads. After aligning with the human genome, we established the molecular identity of both follicles. A total of 617 DEGs were identified, and most play important roles in biological processes, such as the electron transport chain, metabolism, transcriptional/translation activation, biological synthesis, and immune responses, indicating the different contributions of the two kinds of HF to cashmere production. A recent RNA-Seq study (Fan et al., 2015) assembled 13,769 and 12,240 unigenes from primary HFs and secondary HFs, respectively. Of these, 5899 genes were differentially expressed between the anagen and catagen stages in the primary HFs, with 532 genes upregulated and 5367 downregulated. Numerous hair growth genes are expressed in goat HFs, 73 of which exhibit co-upregulation in both HFs during the anagen stage, such as STC2, $V E G F R$, and ROR2 (Fan et al., 2015).

In a previous study, we identified five novel miRNAs in cashmere goat skin and muscle using a comparative genomic approach combined with expression profile analysis ( $\mathrm{Su}$ et al., 2013). Quantitative polymerase chain reactions and sequencing validation demonstrated that three of them were the most highly expressed in February and two in October, indicating that these miRNAs are involved in growth of goat hair and cessation of goat hair production (Su et al., 2013). By using bioinformatics, we investigated the targets of interesting miRNAs, and found that miRNA regulation also contributes to follicular identity and plays an important role in cashmere production. A previous study (Fan et al., 2015) showed that genes that encode keratin and keratin-associated proteins are the most highly expressed, and in the present study, we annotated 20 differentially expressed keratin and keratin-associated protein genes in the primary and secondary follicle transcriptomes.

Hair fiber development is a complicated process that is affected by genetic factors, internal secretion, environment, and nutrition. It is necessary to uncover the molecular basis that underlies the development of HFs and cashmere production. Our results provide useful information about secondary HF development, and are a foundation for future studies.

\section{Conflicts of interest}

The authors declare no conflict of interest.

\section{ACKNOWLEDGMENTS}

Research supported by the National "863" Project (\#2013AA102506), the National Natural Science Foundation of China (\#31201773, \#31260539, \#31272421, and \#31402052), the Excellent Young Scientist Foundation of Inner Mongolia Agricultural University of China (\#2014XYQ-1), the Natural Science Foundation of Inner Mongolia (\#2013MS0414), the Youth Innovation Foundation of Inner Mongolia Academy of Agricultural and Animal Husbandry Sciences (\#2013QNJJM03), and the State Key Laboratory of Genetic Resources and Evolution, Kunming Institute of Zoology, Chinese Academy of Sciences (\#GREKF13-02).

Genetics and Molecular Research 15 (3): gmr.15038589 


\section{REFERENCES}

Andl T, Ahn K, Kairo A, Chu EY, et al. (2004). Epithelial Bmprla regulates differentiation and proliferation in postnatal hair follicles and is essential for tooth development. Development 131: 2257-2268. http://dx.doi.org/10.1242/ dev. 01125

Ashburner M, Ball CA, Blake JA, Botstein D, et al.; The Gene Ontology Consortium (2000). Gene ontology: tool for the unification of biology. Nat. Genet. 25: 25-29. http://dx.doi.org/10.1038/75556

Benjamini Y and Hochberg Y (1995). Controlling the false discovery rate: a practical and powerful approach to multiple testing. J. R. Stat. Soc. B 57: 289-300.

Berger J, Buuveibaatar B and Mishra C (2015). The cashmere connection, biodiversity, and climate: response to von Wehrden et al. 2014. Conserv. Biol. 29: 290-292. http://dx.doi.org/10.1111/cobi.12415

Botchkarev VA and Kishimoto J (2003). Molecular control of epithelial-mesenchymal interactions during hair follicle cycling. J. Investig. Dermatol. Symp. Proc. 8: 46-55.http://dx.doi.org/10.1046/j.1523-1747.2003.12171.x

Botchkarev VA and Paus R (2003). Molecular biology of hair morphogenesis: development and cycling. J. Exp. Zoolog. B Mol. Dev. Evol. 298: 164-180.http://dx.doi.org/10.1002/jez.b.33

Bullard JH, Purdom E, Hansen KD and Dudoit S (2010). Evaluation of statistical methods for normalization and differential expression in mRNA-Seq experiments. BMC Bioinformatics 11: 94.http://dx.doi.org/10.1186/1471-2105-11-94

Dong Y, Xie M, Jiang Y, Xiao N, et al. (2013). Sequencing and automated whole-genome optical mapping of the genome of a domestic goat (Capra hircus). Nat. Biotechnol. 31: 135-141.http://dx.doi.org/10.1038/nbt.2478

Fan YX, Wu RB, Qiao X, Zhang YJ, et al. (2015). Hair follicle transcriptome profiles during the transition from anagen to catagen in Cashmere goat (Capra hircus). Genet. Mol. Res. 14: 17904-17915.http://dx.doi.org/10.4238/2015. December.22.15

Ghosh S and Chan CK (2016). Analysis of RNA-Seq Data Using TopHat and Cufflinks. Methods Mol. Biol. 1374: 339361. http://dx.doi.org/10.1007/978-1-4939-3167-5 18

Ibraheem M, Galbraith H, Scaife J and Ewen S (1994). Growth of secondary hair follicles of the Cashmere goat in vitro and their response to prolactin and melatonin. J. Anat. 185: 135-142.

Keva S (2005). Qualitative and quantitative characteristics of cashmere produced by South African indigenous goats. Master's thesis, University of Pretoria, Pretoria.

Langmead B, Trapnell C, Pop M and Salzberg SL (2009). Ultrafast and memory-efficient alignment of short DNA sequences to the human genome. Genome Biol. 10: R25.http://dx.doi.org/10.1186/gb-2009-10-3-r25

Lefebvre PP, Malgrange B, Staecker H, Moonen G, et al. (1993). Retinoic acid stimulates regeneration of mammalian auditory hair cells. Science 260: 692-695. http://dx.doi.org/10.1126/science.8480180

Luikart G, Gielly L, Excoffier L, Vigne JD, et al. (2001). Multiple maternal origins and weak phylogeographic structure in domestic goats. Proc. Natl. Acad. Sci. USA 98: 5927-5932.http://dx.doi.org/10.1073/pnas.091591198

Marioni JC, Mason CE, Mane SM, Stephens M, et al. (2008). RNA-seq: an assessment of technical reproducibility and comparison with gene expression arrays. Genome Res. 18: 1509-1517.http://dx.doi.org/10.1101/gr.079558.108

Nixon A, Gurnsey M, Betteridge K, Mitchell RJ, et al. (1991). Seasonal hair follicle activity and fibre growth in some New Zealand Cashmere-bearing goats (Capra hircus). J. Zool. (Lond.) 224: 589-598. http://dx.doi. org/10.1111/j.1469-7998.1991.tb03787.x

Rogers G and Bawden C (2009). Improvement of wool production through genetic manipulation. In: Advances in wool technology (Johnson NAG and Russell I, eds.). CRC Press, Boca Raton, 3-21.

Ryder M (1967). Wool fibre shedding in some Merino sheep. Aust. J. Agric. Res. 18: 683-687. http://dx.doi.org/10.1071/ AR9670683

Ryder M (1970). Structure and seasonal change of the coat in Scottish wild goats. J. Zool. 161: 355-361. http://dx.doi. org/10.1111/j.1469-7998.1970.tb04518.x

Schmidt-Ullrich R and Paus R (2005). Molecular principles of hair follicle induction and morphogenesis. BioEssays 27 : 247-261.http://dx.doi.org/10.1002/bies.20184

Schneider MR, Schmidt-Ullrich R and Paus R (2009). The hair follicle as a dynamic miniorgan. Curr. Biol. 19: R132-R142. http://dx.doi.org/10.1016/j.cub.2008.12.005

Stenn KS and Paus R (2001). Controls of hair follicle cycling. Physiol. Rev. 81: 449-494.

Su R, Lv X, Zhao D, Han Z, et al. (2013). Identification and experimental verification of novel microRNA on UniGene in goat. China Anim. Husband. Vet. Med. 40: 118-122.

Trapnell C, Pachter L and Salzberg SL (2009). TopHat: discovering splice junctions with RNA-Seq. Bioinformatics 25: 1105-1111.http://dx.doi.org/10.1093/bioinformatics/btp120

Genetics and Molecular Research 15 (3): gmr.15038589 
Trapnell C, Williams BA, Pertea G, Mortazavi A, et al. (2010). Transcript assembly and quantification by RNA-Seq reveals unannotated transcripts and isoform switching during cell differentiation. Nat. Biotechnol. 28: 511-515. http://dx.doi.org/10.1038/nbt.1621

Viallet JP and Dhouailly D (1994). Retinoic acid and mouse skin morphogenesis. II. Role of epidermal competence in hair glandular metaplasia. Dev. Biol. 166: 277-288. http://dx.doi.org/10.1006/dbio.1994.1314

Wang Z, Gerstein M and Snyder M (2009). RNA-Seq: a revolutionary tool for transcriptomics. Nat. Rev. Genet. 10: 57-63. http://dx.doi.org/10.1038/nrg2484

Wang L, Zhang Y, Zhao M, Wang R, et al. (2015). SNP discovery from transcriptome of cashmere goat skin. Asianaustralas. J. Anim. Sci. 28: 1235-1243. http://dx.doi.org/10.5713/ajas.15.0172

Genetics and Molecular Research 15 (3): gmr.15038589 